\title{
Detection of REST Patterns and Antipatterns: A Heuristics-Based Approach
}

\author{
Francis Palma ${ }^{1,2}$, Johann Dubois ${ }^{1,3}$, Naouel Moha ${ }^{1}$, \\ and Yann-Gaël Guéhéneuc ${ }^{2}$ \\ 1 Département d'informatique, Université du Québec à Montréal, Canada \\ moha.naouel@uqam.ca \\ 2 Ptidej Team, DGIGL, École Polytechnique de Montréal, Canada \\ \{francis.palma, yann-gael.gueheneuc\} @polymtl.ca \\ 3 École supérieure d'informatique, eXia.Cesi, France \\ johann.dubois@viacesi.fr
}

\begin{abstract}
REST (REpresentational State Transfer), relying on resources as its architectural unit, is currently a popular architectural choice for building Web-based applications. It is shown that design patterns - good solutions to recurring design problems - improve the design quality and facilitate maintenance and evolution of software systems. Antipatterns, on the other hand, are poor and counter-productive solutions. Therefore, the detection of REST (anti)patterns is essential for improving the maintenance and evolution of RESTful systems. Until now, however, no approach has been proposed. In this paper, we propose SODA-R (Service Oriented Detection for Antipatterns in REST), a heuristics-based approach to detect (anti)patterns in RESTful systems. We define detection heuristics for eight REST antipatterns and five patterns, and perform their detection on a set of 12 widely-used REST APIs including BestBuy, Facebook, and DropBox. The results show that SODA-R can perform the detection of REST (anti)patterns with high accuracy. We also found that Twitter and DropBox are not well-designed, i.e., contain more antipatterns. In contrast, Facebook and BestBuy are well-designed, i.e., contain more patterns and less antipatterns.
\end{abstract}

Keywords: REST, Antipatterns, Patterns, Design, Heuristics, Detection.

\section{Introduction}

Over the last decade, there is a paradigmatic shift from the traditional standalone software solutions towards the service-oriented paradigm to design, develop, and deploy software systems [1. REST (REpresentational State Transfer) 7] architectural style is simpler and more efficient than the traditional SOAP-based (Simple Object Access Protocol) Web services in publishing and consuming services [18. Thus, RESTful services are gaining an increased attention. Facebook, YouTube, Twitter, and many more companies leverage REST.

However, the increased usage of REST for designing and developing Web-based applications confronts common software engineering challenges. In fact, likewise 
any software system, RESTful systems must evolve to handle new web entities and resources, i.e., meet new business requirements. Even, the changes in underlying technologies or protocols may force the REST APIs to change. All these changes may degrade the design of REST APIs, which may cause the introduction of common poor solutions to recurring design problems - antipatterns - in opposition to design patterns, which are good solutions to the problems that software engineers face while designing and developing RESTful systems. Anti(patterns) might be introduced even in the early design phase of RESTful systems. Antipatterns in RESTful systems not only degrade their design but also make their maintenance and evolution difficult, whereas design patterns facilitate them [3,5, 6].

Forgetting Hypermedia [16] is a common REST antipattern that corresponds to the absence of hypermedia, i.e., links within resource representations. The absence of such links hinders the state transition of RESTful systems and limits the runtime communication between clients and servers. In contrast, Entity Linking [6] - the corresponding design pattern - promotes runtime communication via links provided by the servers within resource representations. By using such hyper-links, the services and consumers can be more autonomous and loosely coupled. For REST APIs, the automatic detection of such (anti)patterns is an important activity by assessing their design (1) to ease their maintenance and evolution and (2) to improve their design quality.

REST (anti)patterns require a concrete detection approach, to support their rigorous analysis, which is still lacking. Despite the presence of several technologyspecific approaches in SCA (Service Component Architecture) and Web services (e.g., 3, 9 11, 13]), they are not applicable for detecting (anti)patterns in REST. Indeed, the key differences between REST architecture and other SOA standards prevents the application of these approaches because: (1) traditional serviceorientation is operations-centric, whereas REST is resources-centric, (2) RESTful services are on top of JSON (or XML) over HTTP, whereas traditional Web services are on top of SOAP over HTTP or JMS (Java Message Service), (3) Web services use WSDL (Web Service Definition Language) as their formal contracts; REST has no standardised contract except the human-readable documentations, (4) traditional services are the set of self-contained software artefacts where operations are denoted using verbs; resources in REST are denoted by nouns and are directlyaccessible objects via URIs, and (5) REST clients use the standard HTTP methods to interact with resources; Web services clients implement separate client-stubs to consume services.

Among many others, the differences discussed above motivate us to propose a new approach, SODA-R (Service Oriented Detection for Antipatterns in REST) to detect (anti)patterns in RESTful systems. SODA-R is supported by an underlying framework, SOFA (Service Oriented Framework for Antipatterns) [9] that supports static and dynamic analyses of service-based systems.

To validate SODA-R, first, we perform a thorough analysis of REST (anti)patterns from the literature $[2,5,6,8,12,16$, and define their detection heuristics. A detection heuristic provides an indication for the presence of certain design issues. For instance, a heuristic "servers should provide entity links in their responses", 
suggests that REST developers need to provide entity links in the responses that REST clients can use. For such case, we define a detection heuristic to check if the response header or body contains any resource location or entity links. Following the defined heuristics, we implement their concrete detection algorithms, apply them on widely used REST APIs, and get the list of REST services detected as (anti)patterns. Our detection results show the effectiveness and accuracy of SODA-R: it can detect five REST patterns and eight REST antipatterns with an average precision and recall of more than of $75 \%$ on 12 REST APIs including BestBuy, Facebook, and DropBox.

Thus, the main contributions in this paper are: (1) the definition of detection heuristics for 13 REST (anti)patterns from the literature, namely [2, 5, 6, 8, 12 , 16, (2) the extension of SOFA framework from its early version [9] to allow the detection of REST (anti)patterns, and, finally, (3) the thorough validation of SODA-R approach with 13 REST (anti)patterns on a set of 12 REST APIs by invoking 115 REST methods from them.

The reminder of the paper is organised as follows. Section 2 briefly describes the contributions from the literature on the specification and detection of SOA (anti)patterns. Section 3 presents our approach SODA-R, while Section 4 presents its validation along with detailed discussions. Finally, Section 5 concludes the paper and sketches the future work.

\section{Related Work}

It is important to design REST (REpresentational State Transfer) APIs of quality for building well-maintainable and evolvable RESTful systems. In the literature, the concept of (anti)patterns are well-recognised as the means to evaluate various design concerns in terms of quality. Despite of the presence of some REST (anti)patterns defined recently by the SOA (Service Oriented Architecture) community, the methods and techniques for their detection are yet to propose.

Indeed, there are few books $[2,5,6$, that discuss a number of REST patterns. In addition, a number of online resources [8, 12, 16] by REST practitioners provide a high-level overview of REST (anti)patterns and discuss how they are introduced by developers at design-time. Beyond those contributions, however, the detection of (anti)patterns require a concrete approach, to support their rigorous analysis, which is still lacking in the current literature.

For instance, Erl in his book [5] discussed $85 \mathrm{SOA}$ patterns related to service design and composition. Erl et al. [6] also explained the REST and RESTful service-orientation, and discussed seven new REST patterns, thus in total, the catalog defines 92 SOA patterns. Daigneau 22 introduced 25 design patterns for SOAP (Simple Object Access Protocol) and RESTful services related to the service interaction, implementation, and evolution. Moreover, various online resources [8, 12, 16] defined a limited number of REST antipatterns related to API design with simple examples. All those books and online resources discussed (1) the solutions to recurring design problems (i.e., patterns) or (2) the bad design practices (i.e., antipatterns), but none of them discussed their detection. 
A few contributions are available on the detection of SOA (anti)patterns for various SOA standards, e.g., SCA (Service Component Architecture) 3, 911] and Web services [13. To the best of our knowledge, the detection of REST (anti)patterns, in the literature deserves yet to receive attention. As a continuous effort to investigate diverse SOA technologies with the goal of detecting REST (anti)patterns, we focus, in this paper, on analysing the REST APIs, both statically and dynamically.

\section{The SODA-R Approach}

We propose the SODA-R approach (Service Oriented Detection for Antipatterns in REST) for the detection of REST (anti)patterns. The steps in SODA-R include:

Step 1. Analysis of Patterns and Antipatterns: This manual step involves analysing the description of REST (anti)patterns to identify the relevant properties that characterise them. We use these properties to define detection heuristics.

Step 2. Detection of Patterns and Antipatterns: This semi-automatic step involves the implementation of detection algorithms based on the heuristics defined in the previous step. Later, we automatically apply these detection algorithms on a set of REST APIs, which return detected (anti)patterns.

The next sections detail the analysis of REST (anti)patterns, the implementation of detection algorithms, and the application of the detection algorithms on REST APIs. The validation of SODA-R is discussed in Section 4

\subsection{Analysis of Patterns and Antipatterns}

For the definition of heuristics, we perform a thorough analysis of REST (anti)patterns by studying their descriptions and examples in the literature [6, 12, 16, 17]. This analysis helps us to identify the static and dynamic properties relevant to each REST (anti)pattern. A static property is a property that is defined on a RESTful service and is obtained statically, i.e., before invoking the REST methods.

A dynamic property, on the other hand, is obtained after making a service call to access a resource and can be found in the request/response headers and bodies, at runtime. For instance, the HTTP request headers Accept and CacheControl and their corresponding values, respectively used to set the resource formats requested by the clients and to set the caching preferences, correspond to dynamic properties. Similarly, the HTTP response headers Location and Status and their corresponding values, respectively used to set the new location by servers and to indicate the current context and status of the action performed by the server on a client request, also correspond to dynamic properties. Table 1 shows the relevant static and dynamic properties for each (anti)pattern, which we use and combine in the following section to define detection heuristics. 
Table 1. Relevant properties of patterns and antipatterns

\begin{tabular}{|l|c|c|}
\hline \multicolumn{1}{|c|}{ REST Antipatterns } & REST Patterns & Properties \\
\hline Breaking Self-descriptiveness & - & request-header fields; response-header fields \\
\hline Forgetting Hypermedia & Entity Linking & http-methods; entity-links; Location \\
\hline Ignoring Caching & Response Caching & Cache-Control; Cache-Control; ETag \\
\hline Ignoring MIME Types & Content Negotiation & Accept; Content-Type \\
\hline Ignoring Status Code & - & http-methods; status; status-code \\
\hline Misusing Cookies & - & Cookie; Set-Cookie \\
\hline Tunnelling Through GET & - & http-method; request-uri \\
\hline Tunnelling Through POST & - & http-method; request-uri \\
\hline- & End-point Redirection & Location; status-code \\
\hline- & Entity Endpoint & end-points; http-methods \\
\hline
\end{tabular}

Detection Heuristics of REST Antipatterns and Patterns: Using thestatic and dynamic properties, we define detection heuristics of REST (anti)patterns. Figures 1 and 2 show the detection heuristics defined for the Forgetting Hypermedia antipattern and the corresponding Entity Linking pattern, respectively.

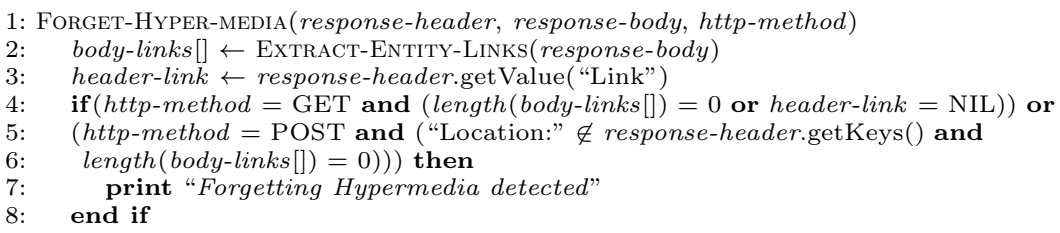

Fig. 1. Heuristic of Forgetting Hypermedia antipattern

Forgetting Hypermedia [16] is a REST antipattern that identifies the absence of entity links in the response body or header. In general, for the HTTP GET requests, the entity links are provided in the response body or header, hence, checking for the absence of links in the response body (i.e., the size of the array containing the entity-links is zero) or the absence of link in the response header is sufficient (line 4, Figure 1). As for the HTTP POST requests, usually the server provides a location in the response header or links in the response body. Therefore, it is sufficient to look for the absence of Location in the response header (line 5, Figure 1) or the absence of links in the response body (line 6, Figure 1) to detect Forgetting Hypermedia antipattern. The corresponding pattern, Entity Linking 6] (Figure 2) refers to a REST service that provides entity links to follow in their response bodies or headers. We put the detection heuristics for the seven other REST antipatterns and four REST patterns on our web site1.

Heuristics are more suitable, in particular for the detection of REST (anti)patterns, because they are more intuitive. Moreover, the engineer's knowledge and experience on REST (anti)patterns play a key role in defining heuristics.

\subsection{Detection of Patterns and Antipatterns}

In this section, we detail the detection of REST (anti)patterns. We show the different implementation and application steps in Figure 3.

\footnotetext{
${ }^{1}$ http://sofa.uqam.ca/soda-r/
} 


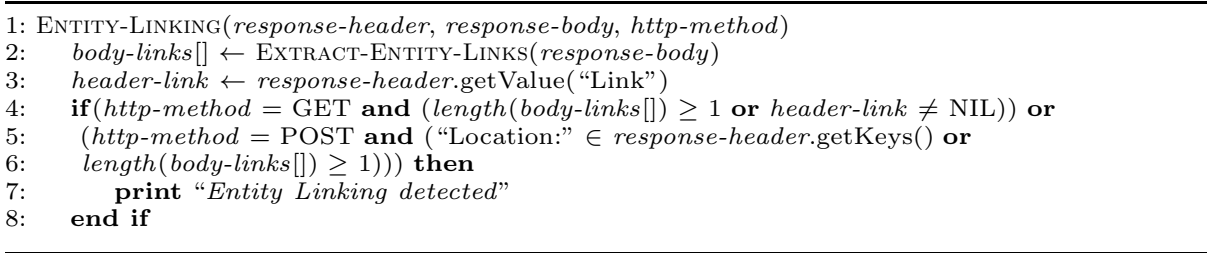

Fig. 2. Heuristic of Entity Linking pattern

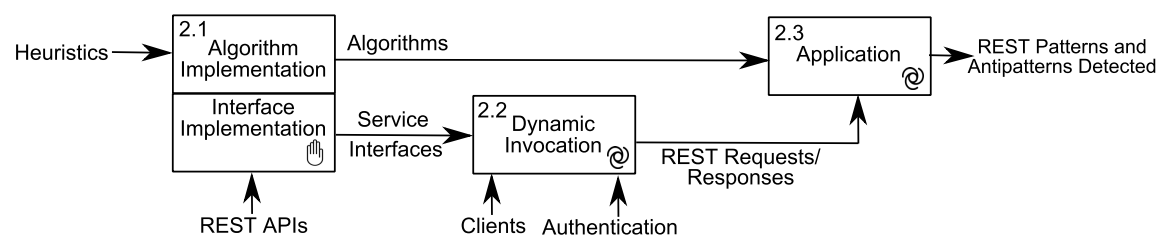

Fig. 3. Steps of the detection of REST (anti)patterns

Step 2.1: Implementation - From the heuristics defined in the previous step (in Section 3.1), we manually implement their corresponding detection algorithms. These algorithms are thus conform to detection heuristics that use and combine static and dynamic properties. We implement also the service interfaces for invoking REST services, and later to analyse their static and dynamic properties. These interfaces written in JAVA contain a set of methods mapped to respective HTTP requests for all REST APIs from their online documentations (see Table 4). The REST API online documentations comprise of (1) a list of resources, (2) a list of actions to perform on these resources, (3) the HTTP requests with entity end-points, and (4) a list of parameters for each HTTP request.

Step 2.2: Dynamic Invocation - After we have JAVA interfaces for REST APIs, we implement the REST clients to invoke each service by providing the correct parameter lists. The REST clients must conform to the API documentations. During the detection time, we dynamically invoke the methods of service interfaces. From REST point of view, invocation of a method refers to performing an action on a resource or on an entity. For some method invocations, clients require to authenticate themselves to the servers. For each authentication process, we need to have a user account to ask for the developer credentials to the server. The server then supplies the user with the authentication details to use every time to make a signed HTTP request. For instance, YouTube and DropBox support OAuth 2.0 authentication protocol to authenticate their clients. At the end of this step, we gather all the requests and responses.

Step 2.3: Application - For the application, we rely on the underlying framework SOFA (Service Oriented Framework for Antipatterns) [9] that enables the analysis of static and dynamic properties specific to REST (anti)patterns. We automatically apply the heuristics in the form of detection algorithms on the requests 
from the clients and responses from the servers, gathered in the previous step. In the end, we obtain a list of detected REST (anti)patterns.

From its initial version in [9], we further developed the SOFA framework to support the detection of REST (anti)patterns. SOFA itself is developed based on the SCA (Service Component Architecture) standard [4] and is composed of several SCA components. SOFA framework uses FraSCAti [15] as its runtime support. We added a new REST Handler SCA component in the framework. The REST Handler component supports the detection of REST (anti)patterns by (1) wrapping each REST API with an SCA component and (2) automatically applying the detection heuristics on the SCA-wrapped REST APIs. This wrapping allows us to introspect each request and response at runtime by using an IntentHandler. The intent handler in FraSCAti is an interceptor that can be applied on a specific service to implement the non-functional features, e.g., transaction or logging. When we invoke a service that uses an IntentHandler, the service call is interrupted and the intent handler is notified by calling the invoke (Intent JoinPoint) method. This interruption of call enables us to introspect the requests and responses of an invoked REST service.

\section{Validation}

In this section, we want to show the robustness of SODA-R approach, accuracy of our detection heuristics, and performance of the implemented algorithms.

\subsection{Hypotheses}

We define three hypotheses to assess the effectiveness of our SODA-R approach. $\mathbf{H}_{1}$. Robustness: The SODA-R approach is robust. This hypothesis claims that our SODA-R approach is assessed rigorously on a large set of REST APIs and with a set of different REST patterns and antipatterns.

$\mathbf{H}_{2}$. Accuracy: The detection heuristics have an average precision of more than $75 \%$ and a recall of $100 \%$, i.e., more than three-quarters of detected (anti)patterns are true positive and we do not miss any existing (anti)patterns. Having a tradeoff between precision and recall, we presume that $75 \%$ precision is acceptable while our objective is to detect all existing (anti)patterns, i.e., $100 \%$ recall. This hypothesis claims the accuracy of the defined detection heuristics and the implemented detection algorithms.

$\mathbf{H}_{3}$. Performance: The implemented algorithms perform with considerably a low detection times, i.e., on an average in the order of seconds. Through this assumption, we support the performance of the implemented detection algorithms.

\subsection{Subjects and Objects}

We define heuristics for eight different REST antipatterns and five REST patterns from the literature. Tables 2 and 3 list those REST antipatterns and patterns 
Table 2. List of eight REST antipatterns

Breaking Self-descriptiveness: REST developers tend to ignore the standardised headers, formats, or protocols and use their own customised ones. This practice shatters the self-descriptiveness or containment of a message header. Breaking the self-descriptiveness also limits the reusability and adaptability of REST resources [16].

Forgetting Hypermedia: The lack of hypermedia, i.e., not linking resources, hinders the state transition for REST applications. One possible indication of this antipattern is the absence of URL links in the resource representation, which typically restricts clients to follow the links, i.e., limits the dynamic communication between clients and servers [16].

Ignoring Caching: REST clients and server-side developers tend to avoid the caching capability due to its complexity to implement. However, caching capability is one of the principle REST constraints. The developers ignore caching by setting Cache-Control: no-cache or no-store and by not providing an ETag in the response header 16.

Ignoring MIME Types: The server should represent resources in various formats, e.g., $x m l, j s o n, p d f$, etc., which may allow clients, developed in diverse languages, a more flexible service consumption. However, the server side developers often intend to have a single representation of resources or rely on their own formats, which limits the resource (or service) accessibility and reusability [16].

Ignoring Status Code: Despite of a rich set of defined application-level status codes suitable for various contexts, REST developers tend to avoid them, i.e., rely only on common ones, namely 200,404 , and 500, or even use the wrong or no status codes. The correct use of status codes from the classes $2 x \boldsymbol{x}, 3 x \boldsymbol{x}, 4 x \boldsymbol{x}$, and $5 \boldsymbol{x} \boldsymbol{x}$ helps clients and servers to communicate in a more semantic manner [16.

Misusing Cookies: Statelessness is another REST principle to adhere-session state in the server side is disallowed and any cookies violate RESTfulness [7]. Sending keys or tokens in the Set-Cookie or Cookie header field to server-side session is an example of misusing cookies, which concerns both security and privacy 16 .

Tunnelling Through GET: Being the most fundamental HTTP method in REST, the GET method retrieves a resource identified by a URI. However, very often the developers rely only on GET method to perform any kind of actions or operations including creating, deleting, or even for updating a resource. Nevertheless, HTTP GET is an inappropriate method for any actions other than accessing a resource, and does not match its semantic purpose, if improperly used 16.

Tunnelling Through POST: This anti-pattern is very similar to the previous one, except that in addition to the URI the body of the HTTP POST request may embody operations and parameters to apply on the resource. The developers tend to depend only on HTTP POST method for sending any types of requests to the server including accessing, updating, or deleting a resource. In general, the proper use of HTTP POST is to create a server-side resource [16].

collected from the literature, mainly $6,8,12,16,17]$. In Tables 2 and 3 , we put the relevant properties for each antipattern and pattern in bold-italics.

As for the objects in our experiment, we use some widely-used and popular REST APIs for which their underlying HTTP methods, service end-points, and authentication details are well documented online. Large companies like Facebook or YouTube provide self-contained documentations with good example sets. Table 4 lists the 12 REST APIs that we analysed in our experiment.

\subsection{Validation Process}

Through the implemented clients, we invoked a total set of 115 methods from the service interfaces to access resources and received the responses from servers. Then, we applied the detection algorithms on the REST requests and responses and reported any existing patterns or antipatterns using our SOFA framework. We manually validated the detection results to identify the true positives and 
Table 3. List of five REST patterns

Content Negotiation: This pattern supports alternative resource representations, e.g., in json, $\boldsymbol{x m l}, \boldsymbol{p d f}$, etc. so that the service consuming becomes more flexible with high reusability. Servers can provide resources in any standard format requested by the clients. This pattern is applied via standard HTTP media types and adhere to service loose coupling principle. If not applied at all, this turns into Ignoring MIME Types antipattern 6].

End-point Redirection: The redirection feature over the Web is supported by this pattern, which also plays a role as the means of service composition. To redirect clients, servers send a new location to follow with one of the status code among 301, 302, 307, or 308. The main benefit of this pattern is-an alternative service remains active even if the requested service end-point is not sound [6].

Entity Linking: This pattern enables runtime communication via links provided by the server in the response body or via Location: in the response header. By using hyper-links, the servers and clients can be loosely coupled, and the clients can automatically find the related entities at runtime. If not properly applied, this pattern turns into Forgetting Hypermedia antipattern 6].

Entity Endpoint: Services with single end-points are too coarse-grained. Usually, a client requires at least two identifiers: (1) a global for the service itself and (2) a local for the resource or entity managed by the service. By applying this pattern, i.e., using multiple end-points, each entity (or resource) of the incorporating service can be uniquely identified and addressed globally [12].

Response Caching: Response caching is a good practice to avoid sending duplicate requests and responses by caching all response messages in the local client machine. In opposed to Ignoring Caching antipattern, the Cache-Control: is set to any value other than no-cache and no-store, or an ETag is used along with the status code 304 [6].

Table 4. List of 12 REST APIs and their online documentations.

\begin{tabular}{|l|l|}
\hline REST APIs & \multicolumn{1}{|c|}{ Online Documentations } \\
\hline Alchemy & alchemyapi.com/api/ \\
\hline BestBuy & bbyopen.com/developer/ \\
\hline Bitly & dev.bitly.com/api.html \\
\hline CharlieHarvey & charlieharvey.org.uk/about/api/ \\
\hline DropBox & dropbox.com/developers/core/docs/ \\
\hline Facebook & developers.facebook.com/docs/graph-api/ \\
\hline Musicgraph & developer.musicgraph.com/api-docs/overview/ \\
\hline Ohloh & github.com/blackducksw/ohloh_api/ \\
\hline TeamViewer & integrate.teamviewer.com/en/develop/documentation/ \\
\hline Twitter & dev.twitter.com/docs/api/ \\
\hline YouTube & developers.google.com/youtube/v3/ \\
\hline Zappos & developer.zappos.com/docs/api-documentation/ \\
\hline
\end{tabular}

to find false negatives. The validation was performed by two professionals who have knowledge on REST and were not part of the implementation and experiment. We provided them the descriptions of REST (anti)patterns and the sets of all requests and responses collected during the service invocations. We used precision and recall to measure our detection accuracy. Precision concerns the ratio between the true detected (anti)patterns and all detected (anti)patterns. Recall is the ratio between the true detected (anti)patterns and all existing true (anti)patterns.

\subsection{Results}

Table[5presents detailed detection results for the eight REST antipatterns and five REST patterns. The table reports the (anti)patterns in the first column followed 


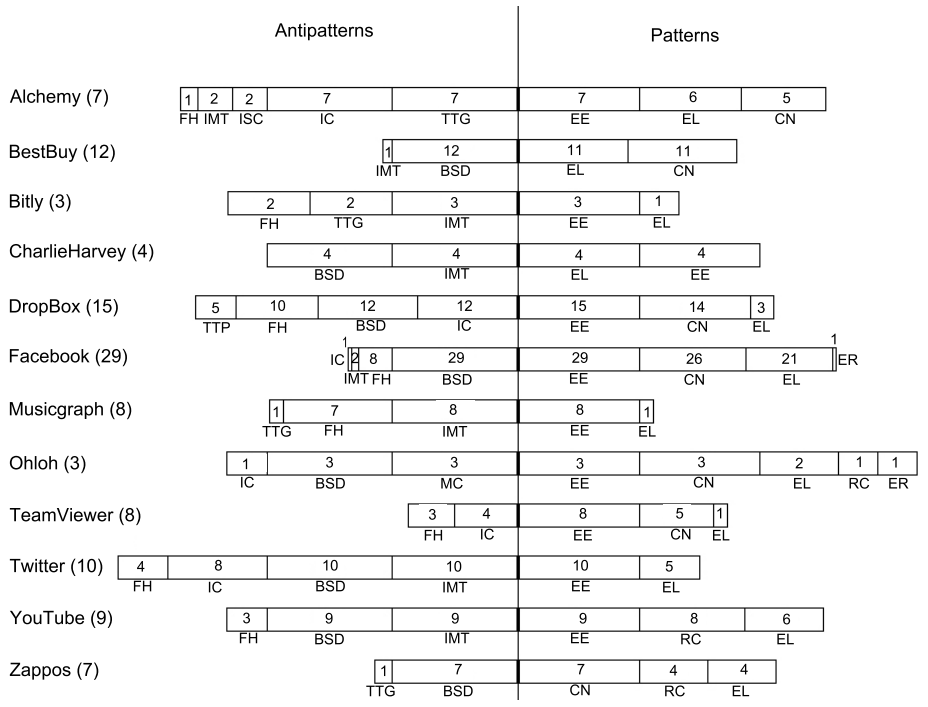

Fig. 4. Bar-plots of the detection results for eight antipatterns and five patterns. (APIs are followed by the number of method invocations in parentheses. The acronyms correspond to the (anti)pattern name abbreviation and the numbers represent their detected instances.)

by the analysed REST APIs in the following twelve columns. For each REST API and for each (anti)pattern, we report: (1) the total number of validated true positives with respect to the total detected (anti)patterns by our algorithms, i.e., the precision, in the first row and (2) the total number of detected true positives with respect to the total existing true positives, i.e., the recall, in the following row. The last two columns show, for all APIs, the average precisionrecall and the total detection time for each (anti)pattern. The detailed results on all the test cases, e.g., 115 methods from 12 REST APIs, are available on our web site 1 .

\subsection{Overview on the Results}

Figure 4 shows the bar-plots of the detection results for the eight antipatterns and five patterns on the 12 REST APIs.

REST developers are most likely to use their own header fields, data formats, and protocols, which limit the comprehension and reusability of REST APIs. For example, among more than 80 instances of detected Breaking Self-descriptiveness (BSD) antipattern: Facebook (29 instances), DropBox (12 instances), and BestBuy (12 instances) were mostly using customised header fields, data formats, and protocols. Also, Forgetting Hypermedia (FH) antipattern was detected in Facebook (8 instances) and DropBox (10 instances) APIs. Moreover, Ignoring MIME Types (IMT) antipattern was detected in Twitter (10 instances) and YouTube (9 instances) APIs. Among the less frequent antipatterns, Ignoring Status Code 
(ISC, 2 instances) and Misusing Cookies (MC, 3 instances) were not significantly observed among the 115 tested methods.

As for REST patterns, Content Negotiation (CN, 70 instances) and Entity Linking (EL, 62 instances) were most frequently applied by REST developers. Content Negotiation pattern supports the ability to represent REST resources in diverse formats (implemented by REST developers) as requested by the clients. Entity Linking pattern facilitates clients to follow links provided by the servers. Furthermore, some APIs also applied Response Cashing (RC, 13 instances) and End-point Redirection (ER, 2 instances) patterns.

Overall, REST APIs that follow patterns tend to avoid corresponding antipatterns and vice-versa. For example: BestBuy and Facebook are found involved respectively in 0 and 8 instances of Forgetting Hypermedia antipattern; however, these APIs are involved in 11 and 21 corresponding Entity Linking pattern. Moreover, DropBox, Alchemy, YouTube, and Twitter APIs had 27 instances of Ignoring Caching antipattern, but they were involved in 8 instances of the corresponding Response Cashing pattern. Finally, we found Facebook, DropBox, BestBuy, and Zappos APIs involved in only 3 instances of Ignoring MIME Types antipattern, which conversely are involved in more than 55 instances of corresponding Content Negotiation pattern.

In general, among the 12 analysed REST APIs with 115 methods tested and eight antipatterns, we found Twitter (32 instances of four antipatterns), DropBox (40 instances of four antipatterns), and Alchemy (19 instances of five antipatterns) are more problematic, i.e., contain more antipatterns than others (see Figure 4). On the other hand, considering the five REST patterns, we found Facebook (49 instances of four patterns), BestBuy (22 instances of two patterns), and YouTube (15 instances of three patterns) are well designed, i.e., involve more patterns than others (see Figure 4).

\subsection{Details of the Results}

In this section, we discuss three detection results in detail, obtained in our experiment as presented in Table 5 .

REST developers tend to rely on their own customised headers, formats, and protocols, and thus introduce Breaking Self-descriptiveness antipattern. The analysis on the 12 REST APIs shows that developers used non-standard header fields and protocols in most APIs including BestBuy, DropBox, Facebook, and Twitter. For example, Facebook used $\mathrm{x}-\mathrm{fb}$-debug and $\mathrm{x}-\mathrm{f} \mathrm{b}-\mathrm{rev}$ header fields, which are mainly used to track a request id for their internal bug management purpose. Similarly, we found DropBox using the $\mathrm{x}$-dropbox-request-id and Twitter using $\mathrm{x}$-tfe-logging-request-* and $\mathrm{x}$-xss-protection header fields. In general, the designers and implementers often distinguish the standardised and non-standardised header members by prefixing their names with "x-" (a.k.a. eXperimental). Indeed, the "x-" convention was highly discouraged by the Internet Society in RFC822 14. The manual validation reveals that all our detection was true positives and we reported all existing non-standard header fields and protocols, except two in DropBox where the manual validation considered 
Table 5. Detection results of the eight REST antipatterns and five REST patterns obtained by applying detection algorithms on the 12 REST APIs (numbers in the parentheses show total test methods for each API).

\begin{tabular}{|c|c|c|c|c|c|c|c|c|c|c|c|c|c|c|c|c|}
\hline 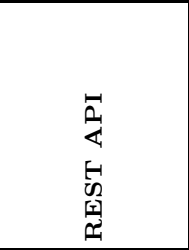 & 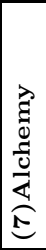 & 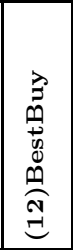 & 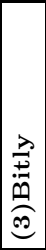 & 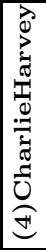 & 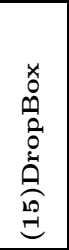 & 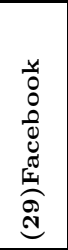 & 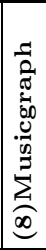 & $\begin{array}{l}\frac{1}{0} \\
\frac{1}{1} \\
0 \\
\underbrace{n}\end{array}$ & 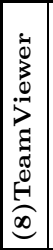 & 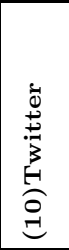 & 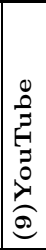 & 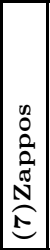 & 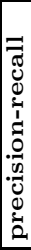 & 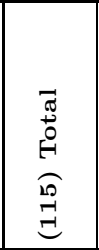 & 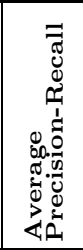 & 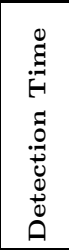 \\
\hline \multicolumn{17}{|c|}{ REST Antipatterns } \\
\hline \multirow{2}{*}{$\begin{array}{l}\text { Breaking Self- } \\
\text { descriptiveness }\end{array}$} & $0 / 0$ & $12 / 12$ & $0 / 0$ & $4 / 4$ & $12 / 12$ & $29 / 29$ & $0 / 0$ & $3 / 3$ & $0 / 0$ & $10 / 10$ & $9 / 9$ & $7 / 7$ & $\mathrm{p}$ & $86 / 86$ & \multirow{2}{*}{\begin{tabular}{|c|}
$100 \%$ \\
$998.21 \%$ \\
\end{tabular}} & \multirow[b]{2}{*}{$21.31 \mathrm{~s}$} \\
\hline & $\dddot{0} \%$ & $12 / 12$ & $0 \%$ & $\dddot{4} / 4$ & $12 / 14$ & $29 / 29$ & $0 \%$ & $3 / 3$ & $0 \%$ & $10 / 10$ & $\dddot{9} \%$ & $7 / 7$ & $\mathrm{r}$ & $86 / 88$ & & \\
\hline \multirow{2}{*}{$\begin{array}{l}\text { Forgetting } \\
\text { Hypermedia }\end{array}$} & $1 / 1$ & $0 / 0$ & $2 / 2$ & $0 / 0$ & $9 / 10$ & $8 / 8$ & $7 / 7$ & $0 / 0$ & $3 / 3$ & $4 / 4$ & $2 / 3$ & $0 / 0$ & $p$ & $36 / 38$ & $94.58 \%$ & \multirow{2}{*}{$19.54 \mathrm{~s}$} \\
\hline & $1 / 1$ & $0 \%$ & $2 / 2$ & $0 \%$ & $9 / 9$ & $8 / 8$ & $7 / 7$ & $0 \%$ & $3 / 3$ & 474 & $2 / 2$ & $0 \%$ & $\mathrm{r}$ & $36 / 36$ & $100 \%$ & \\
\hline \multirow{2}{*}{$\begin{array}{l}\text { Ignoring } \\
\text { Caching }\end{array}$} & $7 / 7$ & $0 / 0$ & $0 / 0$ & $0 / 0$ & $12 / 12$ & $1 / 1$ & $0 / 0$ & $1 / 1$ & $4 / 4$ & $8 / 8$ & $0 / 0$ & $0 / 0$ & $p$ & $33 / 33$ & $100 \%$ & \multirow{2}{*}{$18.99 \mathrm{~s}$} \\
\hline & $7 / 7$ & $0 \% 0$ & $0 / 0$ & $0 \% 0$ & $12 / 12$ & 1/1 & $0 \%$ & $1 / 1$ & $4 / 4$ & $8 / 8$ & $0 \% 0$ & $0 \%$ & $\mathrm{r}$ & $33 / 33$ & $100 \%$ & \\
\hline \multirow{2}{*}{$\begin{array}{l}\text { Ignoring } \\
\text { MIME Types }\end{array}$} & $2 / 2$ & $1 / 1$ & $3 / 3$ & $4 / 4$ & $0 / 0$ & $2 / 2$ & $8 / 8$ & $0 / 0$ & $0 / 0$ & $10 / 10$ & $9 / 9$ & $0 / 0$ & $p$ & $39 / 39$ & $100 \%$ & \multirow[b]{2}{*}{$19.39 \mathrm{~s}$} \\
\hline & $2 / 2$ & $1 / 1$ & $3 / 3$ & $\dddot{4} / 4$ & $0 / 0$ & $2 / 2$ & $8 / 8$ & $0 \% 0$ & $0 \%$ & $10 / 10$ & $9 / 9$ & $0 \% 0$ & $\mathrm{r}$ & $39 / 39$ & $100 \%$ & \\
\hline \multirow{2}{*}{$\begin{array}{l}\text { Ignoring } \\
\text { Status Code }\end{array}$} & $1 / 2$ & $0 / 0$ & $0 / 0$ & $0 / 0$ & $0 / 0$ & $0 / 0$ & $0 / 0$ & $0 / 0$ & $0 / 0$ & $0 / 0$ & $0 / 0$ & $0 / 0$ & $\mathrm{p}$ & $1 / 2$ & $50 \%$ & \multirow{2}{*}{$21.22 \mathrm{~s}$} \\
\hline & $1 / 2$ & $0 \% 0$ & $0 / 1$ & $\dddot{0} \%$ & $0 / 0$ & $0 \%$ & $0 \% 0$ & $0 \% 0$ & $0 \% 0$ & $0 \%$ & $0 \% 0$ & $0 \%$ & $\mathrm{r}$ & $1 / 3$ & $25 \%$ & \\
\hline \multirow{2}{*}{$\begin{array}{l}\text { Misusing } \\
\text { Cookies }\end{array}$} & $0 / 0$ & $0 / 0$ & $0 / 0$ & $0 / 0$ & $0 / 0$ & $0 / 0$ & $0 / 0$ & $3 / 3$ & $0 / 0$ & $0 / 0$ & $0 / 0$ & $0 / 0$ & $p$ & $3 / 3$ & $100 \%$ & \multirow[b]{2}{*}{$19.1 \mathrm{~s}$} \\
\hline & $0 \% 0$ & $0 \%$ & $0 \% 0$ & $0 \% 0$ & $0 \% 0$ & $0 \%$ & $0 \% 0$ & $3 / 3$ & $0 \%$ & $0 \%$ & $0 \% 0$ & $0 \% 0$ & $\mathrm{r}$ & $3 / 3$ & $100 \%$ & \\
\hline \multirow{2}{*}{$\begin{array}{l}\text { Tunnelling } \\
\text { Through GET }\end{array}$} & $5 / 7$ & $0 / 0$ & $0 / 2$ & $0 / 0$ & $0 / 0$ & $0 / 0$ & $0 / 1$ & $0 / 0$ & $0 / 0$ & $0 / 0$ & $0 / 0$ & $0 / 1$ & $p$ & $5 / 11$ & $17.86 \%$ & \multirow{2}{*}{$28.26 \mathrm{~s}$} \\
\hline & $5 / 5$ & $0 \%$ & $0 \% 0$ & $0 \% 0$ & $0 / 0$ & $0 / 0$ & $0 \% 0$ & $0 \% 0$ & $0 \%$ & $0 / 0$ & $0 \% 0$ & $0 \% 0$ & $\mathrm{r}$ & $5 / 5$ & $100 \%$ & \\
\hline \multirow{2}{*}{$\begin{array}{l}\text { Tunnelling } \\
\text { Through POST }\end{array}$} & $0 / 0$ & $0 / 0$ & $0 / 0$ & $0 / 0$ & $5 / 5$ & $0 / 0$ & $0 / 0$ & $0 / 0$ & $0 / 0$ & $0 / 0$ & $0 / 0$ & $0 / 0$ & $p$ & $5 / 5$ & $100 \%$ & \multirow{2}{*}{$28.64 \mathrm{~s}$} \\
\hline & $0 \%$ & $0 / 0$ & $0 \%$ & $0 \%$ & $5 / 5$ & $0 / 0$ & $0 / 0$ & $0 / 0$ & $0 \%$ & $0 / 0$ & $0 / 0$ & $0 \% 0$ & $\mathrm{r}$ & $5 / 5$ & $100 \%$ & \\
\hline \multicolumn{17}{|c|}{ REST Patterns } \\
\hline \multirow{2}{*}{$\begin{array}{l}\text { Content } \\
\text { Negotiation }\end{array}$} & $5 / 5$ & $11 / 11$ & $0 / 0$ & $0 / 0$ & $14 / 14$ & $26 / 26$ & $0 / 0$ & $3 / 3$ & $5 / 5$ & $0 / 0$ & $0 / 0$ & $7 / 7$ & $p$ & $71 / 71$ & $100 \%$ & \multirow{2}{*}{$19.63 \mathrm{~s}$} \\
\hline & $5 \% 5$ & i1i1i & $0 \%$ & $0 \%$ & $14 / 14$ & $26 / 26$ & $0 \%$ & $3 / 3$ & $5 / 5$ & $0 \%$ & $0 \% 0$ & $7 / 7$ & $\mathrm{r}$ & $71 / 71$ & $100 \%$ & \\
\hline \multirow{2}{*}{$\begin{array}{l}\text { Entity } \\
\text { Linking }\end{array}$} & $6 / 6$ & $11 / 11$ & $1 / 1$ & $4 / 4$ & $3 / 3$ & $21 / 21$ & $1 / 1$ & $2 / 2$ & $1 / 1$ & $5 / 5$ & $6 / 6$ & $4 / 4$ & $p$ & $65 / 65$ & $100 \%$ & \multirow{2}{*}{$19.90 \mathrm{~s}$} \\
\hline & $6 / 6$ & 111/11 & $1 / 1$ & $\dddot{4} / 4$ & $3 / 3$ & $21 / 21$ & $1 / 1$ & $2 / 2$ & $1 / 1$ & $5 \% 5$ & $6 / 7$ & $4 / 4$ & $\mathrm{r}$ & $65 / 66$ & $98.81 \%$ & \\
\hline \multirow{2}{*}{$\begin{array}{l}\text { End-point } \\
\text { Redirection }\end{array}$} & $0 / 0$ & $0 / 0$ & $0 / 0$ & $0 / 0$ & $0 / 0$ & $1 / 1$ & $0 / 0$ & $1 / 1$ & $0 / 0$ & $\begin{array}{ll}0 / 0 \\
0.10\end{array}$ & $0 / 0$ & $0 / 0$ & $\mathrm{p}$. & $2 / 2$ & $100 \%$ & \multirow{2}{*}{$20.36 \mathrm{~s}$} \\
\hline & $0 \% 0$ & $0 \% 0$ & $0 / 0$ & $0 \%$ & $0 / 0$ & $1 / 1$ & $0 \% 0$ & $1 / 1$ & $0 \%$ & $0 / 0$ & $0 / 0$ & $0 / 0$ & $\mathrm{r}$ & $2 / 2$ & $100 \%$ & \\
\hline Entity & $1 / 1$ & $0 / 0$ & $1 / 1$ & $1 / 1$ & $1 / 1$ & $1 / 1$ & $1 / 1$ & $1 / 1$ & $1 / 1$ & $1 / 1$ & $1 / 1$ & $0 / 0$ & $p$ & $10 / 10$ & $100 \%$ & \\
\hline Endpoint & $1 \% 1$ & $0 \% 0$ & $1 / 1$ & $1 / 1$ & $1 / 1$ & $1 \% 1$ & $1 / 1$ & $1 / 1$ & $1 / 1$ & $1 / 1$ & $1 / 1$ & $0 \% 0$ & $\mathrm{r}$ & $10 / 10$ & $100 \%$ & .005 \\
\hline Response & $0 / 0$ & $0 / 0$ & $0 / 0$ & $0 / 0$ & $0 / 0$ & $\begin{array}{ll}0 / 0 \\
0 / 0\end{array}$ & $0 / 0$ & $1 / 1$ & $0 / 0$ & $\begin{array}{ll}0 / 0 \\
0.10\end{array}$ & $8 / 8$ & $4 / 4$ & $\mathrm{p}$ & $13 / 13$ & $100 \%$ & \\
\hline Caching & $0 \%$ & $0 \%$ & $0 \%$ & $0 \%$ & $0 \% 0$ & $0 \%$ & $0 \% 0$ & $1 / 1$ & $0 \%$ & $0 \%$ & $8 / 8$ & $4 / 4$ & $\mathrm{r}$ & $13 / 13$ & $100 \%$ & \\
\hline & & & & & ag & & & & & & & & $p$ & \begin{tabular}{|c|}
$369 / 378$ \\
$360 / 378$
\end{tabular} & \begin{tabular}{l}
$89.42 \%$ \\
\hdashline $04 \%$
\end{tabular} & $21.43 \mathrm{~s}$ \\
\hline
\end{tabular}

them as non-standard practice. This leads to the precision of $100 \%$ and the recall of $98.21 \%$ for this detection.

Any RESTful interaction is driven by hypermedia - by which clients interact with application servers via URL links provided by servers in resource representations [7]. The absence of such interaction pattern is known as Forgetting Hypermedia antipattern [16, which was detected in eight APIs, namely Bitly, DropBox, Facebook, and so on (see Table 5). Among the 115 methods tested, we found 38 instances of this antipattern. Moreover, REST APIs that do not have this antipattern well applied the corresponding Entity Linking pattern [6], e.g., Alchemy, BestBuy, and Ohloh, which is a good practice. This observation suggests that, in practice, developers sometimes do not provide hyper-links in resource representations. As for the validation, 36 instances of Forgetting Hypermedia antipattern were manually validated; therefore, we have an average precision of $94.58 \%$ and a recall of $100 \%$. For Entity Linking pattern, the manual validation confirmed 66 instances whereas we detected a total of 65 instances, 
all of which were true positives. Thus, we had an average precision of $100 \%$ and a recall of $98.81 \%$.

Caching helps developers implementing high-performance and scalable REST services by limiting repetitive interactions, which if not properly applied violates one of the six REST principles [7]. REST developers widely ignore the caching capability by using Pragma: no-cache or Cache-Control: no-cache header in the requests, which forces the application to retrieve duplicate responses from servers. This bad practice is known as Ignoring Caching antipattern [16. In contrast, the corresponding pattern, Response Caching [6] supports response cacheability. We detected six REST APIs that explicitly avoid caching capability, namely Alchemy, DropBox, Ohloh, and so on (see Table 5). On the other hand, cacheability is supported by YouTube and Zappos, which were detected as Response Caching patterns. The manual analysis of requests and responses also confirmed these detections, and we had an average precision and recall of $100 \%$.

\subsection{Discussion on the Hypotheses}

In this section, we discuss the hypotheses defined in Section 4.1

$\mathbf{H}_{1}$. Robustness: To validate the SODA-R approach, we performed experiment on 12 REST APIs including well-known Facebook, BestBuy, DropBox, Twitter, and YouTube. We analysed 115 methods in the form of HTTP requests from these APIs and applied detection algorithms of eight common REST antipatterns and five REST patterns on them. For each request among 115, we analysed individual request headers and bodies, and the corresponding response headers and bodies. With such an extensive evaluation and validation, we support our first hypothesis on the robustness of our SODA-R approach.

$\mathbf{H}_{2}$. Accuracy: As shown in Table 5, we obtained an average recall of $94 \%$ and an average precision of $89.42 \%$ on all REST APIs and for all test methods. The precision ranges from $17.86 \%$ to $100 \%$, while we obtained a recall between $25 \%$ and $100 \%$ for all REST (anti)patterns. Thus, with an average precision of $89.42 \%$ and a recall of $94 \%$, we can positively support our second hypothesis on the accuracy of our defined heuristics and implemented detection algorithms.

$\mathbf{H}_{3}$. Performance: The total required time includes: (i) the execution time, i.e., sending REST requests and receiving REST responses (ranges from 19.1s to $24.55 \mathrm{~s}$ ) and (ii) the time required to apply and run the detection algorithms on the requests and responses (ranges from 0.004s to 4.312s). Each row in Table 5 (last column) reports the total required detection time for a pattern or an antipattern, which varies from 19.1s to 28.64s. We performed our experiments on an Intel Core-i7 with a processor speed of $2.50 \mathrm{GHz}$ and $6 \mathrm{~GB}$ of memory. The detection time is comparatively very low (on an average $3 \%$ of the total required time) than the execution time. With a low average detection time of $21.43 \mathrm{~s}$, we can positively support our third hypothesis on performance. 


\subsection{Threats to Validity}

As future work, we plan to generalise our findings to other REST APIs. However, we tried to minimise the threat to the external validity of our results by performing experiments on 12 REST APIs by invoking and testing 115 methods from them. The detection results may vary based on the heuristics defined for the REST (anti)patterns. To minimise the threats to the Internal validity, we made sure that every invocation receives responses from servers with the correct request URI, and the client authentication done while necessary. Moreover, we tested all the major HTTP methods in REST, i.e., GET, DELETE, PUT, and POST on resources to minimise the threat to the internal validity. Engineers may have different views and different levels of expertise on REST (anti)patterns, which may affect the definition of heuristics. We attempted to lessen the threat to construct validity by defining the heuristics after a thorough review of existing literature on the REST (anti)patterns. We also involved two professionals in the intensive validation of the results. Finally, the threats to reliability validity concerns the possibility of replicating this study. To minimise this threat, we provide all the details required to replicate the study, including the heuristics, client requests, and server responses on our web site ${ }^{1}$ ?

\section{Conclusion and Future Work}

REST (REpresentational State Transfer) is now a popular architectural style for building Web-based applications. REST developers may apply design patterns or introduce antipatterns. These REST patterns and antipatterns may respectively: (1) facilitate and hinder semantically richer communications between clients and servers, or (2) ease and cause difficult maintenance and evolution.

This paper presented the SODA-R approach (Service Oriented Detection for Antipatterns in REST) to define detection heuristics and detect REST (anti)patterns in REST APIs. The detection of (anti)patterns in REST APIs requires an in-depth analysis of their design, invocation, and authentication. We applied SODA-R to define the detection heuristics of five common REST patterns and eight REST antipatterns. Using an extended SOFA framework (Service Oriented Framework for Antipatterns), we performed an extensive validation with 13 REST (anti)patterns. We analysed 12 REST APIs and tested 115 methods, and showed the accuracy of SODA-R with an average precision of $89.42 \%$ and recall of $94 \%$.

In future work, we want to replicate SODA-R on other REST APIs and methods with more REST (anti)patterns. We also intend to enrich the catalog of antipatterns and patterns by thoroughly investigating a large set of REST APIs.

Acknowledgements. The authors thank Abir Dilou for initiating the study. This study is supported by NSERC (Natural Sciences and Engineering Research Council of Canada) and FRQNT research grants. 


\section{References}

1. Bennett, K., Layzell, P., Budgen, D., Brereton, P., Macaulay, L., Munro, M.: Service-based Software: The Future for Flexible Software. In: Proceedings of Seventh Asia-Pacific Software Engineering Conference, pp. 214-221 (2000)

2. Daigneau, R.: Service Design Patterns: Fundamental Design Solutions for SOAP/WSDL and RESTful Web Services. Addison-Wesley (November 2011)

3. Demange, A., Moha, N., Tremblay, G.: Detection of SOA Patterns. In: Basu, S., Pautasso, C., Zhang, L., Fu, X. (eds.) ICSOC 2013. LNCS, vol. 8274, pp. 114-130. Springer, Heidelberg (2013)

4. Edwards, M.: Service Component Architecture (SCA). OASIS, USA (April 2011)

5. Erl, T.: SOA Design Patterns. Prentice Hall PTR (January 2009)

6. Erl, T., Carlyle, B., Pautasso, C., Balasubramanian, R.: SOA with REST: Principles, Patterns \& Constraints for Building Enterprise Solutions with REST. The Prentice Hall Service Technology Series from Thomas Erl. (2012)

7. Fielding, R.T.: Architectural Styles and the Design of Network-based Software Architectures. PhD thesis (2000)

8. Fredrich, T.: RESTful Service Best Practices: Recommendations for Creating Web Services (May 2012)

9. Moha, N., Palma, F., Nayrolles, M., Conseil, B.J., Guéhéneuc, Y.-G., Baudry, B., Jézéquel, J.-M.: Specification and Detection of SOA Antipatterns. In: Liu, C., Ludwig, H., Toumani, F., Yu, Q. (eds.) Service Oriented Computing. LNCS, vol. 7636, pp. 1-16. Springer, Heidelberg (2012)

10. Nayrolles, M., Moha, N., Valtchev, P.: Improving SOA Antipatterns Detection in Service Based Systems by Mining Execution Traces. In: 20th Working Conference on Reverse Engineering, pp. 321-330 (October 2013)

11. Palma, F., Nayrolles, M., Moha, N., Guéhéneuc, Y.G., Baudry, B., Jézéquel, J.M.: SOA Antipatterns: An Approach for their Specification and Detection. International Journal of Cooperative Information Systems 22(04) (2013)

12. Pautasso, C.: Some REST Design Patterns (and Anti-Patterns) (October 2009), http://www.jopera.org/node/442

13. Penta, M.D., Santone, A., Villani, M.L.: Discovery of SOA Patterns via Model Checking. In: 2nd International Workshop on Service Oriented Software Engineering: In Conjunction with the 6th ESEC/FSE Joint Meeting, IW-SOSWE 2007, pp. 8-14. ACM, New York (2007)

14. RFC2822: Internet Message Format by Internet Engineering Task Force. Technical report (2001)

15. Seinturier, L., Merle, P., Rouvoy, R., Romero, D., Schiavoni, V., Stefani, J.B.: A Component-Based Middleware Platform for Reconfigurable Service-Oriented Architectures. Software: Practice and Experience 42(5), 559-583 (2012)

16. Tilkov, S.: REST Anti-Patterns (July 2008), http://www. infoq.com/articles/rest-anti-patterns

17. Tilkov, S.: RESTful Design: Intro, Patterns, Anti-Patterns (December 2008), http://www.devoxx.com/

18. Vinoski, S.: Serendipitous Reuse. IEEE Internet Computing 12(1), 84-87 (2008) 